\title{
Chronobiology, excessive daytime sleepiness and depression: Is there a link?
}

\author{
Sarah Laxhmi Chellappa ${ }^{\mathrm{a}, \mathrm{c}}$, Carmen Schröder ${ }^{\mathrm{b}}$, Christian Cajochen $^{\mathrm{c}, *}$ \\ ${ }^{a}$ The CAPES Foundation/Ministry of Education of Brazil, Caixa Postal 365, CEP. 70359-970, Brasilia-DF, Brazil \\ ${ }^{\mathrm{b}}$ Department of Psychiatry and Behavioural Sciences, Stanford University School of Medicine, 401 Quarry Road, Stanford, CA 94305-5550, USA \\ ${ }^{\mathrm{c}}$ Centre for Chronobiology, Psychiatric University Clinics, Wilhelm Kleinstrasse 27, CH-4025 Basel, Switzerland
}

Received 5 February 2008; received in revised form 30 April 2008; accepted 2 May 2008

Available online 27 September 2008

\begin{abstract}
The complaint of excessive daytime sleepiness (EDS), commonly encountered in clinical practice, may arise from a variety of psychiatric disorders, most importantly depression. Even though EDS frequently leads depressed patients to seek medical assistance, it is commonly under-evaluated and under-diagnosed. Therefore, a comprehensive understanding and management of EDS is essential in the clinical assessment of depression. Within a theoretical framework, a chronobiological approach may shed new light on the complex interaction of EDS and depression. In this review, studies on EDS and depression are summarized and discussed within the context of circadian and sleep regulatory mechanisms. Furthermore, potential chronobiological therapeutic strategies are proposed to address some of the unmet needs in the treatment of EDS and depression.
\end{abstract}

(C) 2008 Elsevier B.V. All rights reserved.

Keywords: Daytime sleepiness; Sleep disorders; Depressive disorders; Light therapy; Sleep deprivation; Circadian rhythm

\section{Introduction}

Excessive daytime sleepiness (EDS) is a tendency to fall asleep despite volitional attempts to remain alert. Chronic sleep loss and/or poor sleep quality are the major underlying reasons for EDS. They occur in numerous sleep disorders, such as obstructive sleep apnea and narcolepsy, as well as in psychiatric disorders, particularly depression. Approximately $80 \%$ of depressive states are associated with comorbid insomnia, related or not to EDS [1]. Depressed patients exhibit a plethora of complaints related to daytime energy levels. However, even though sleep quality and quantity are very often substantially decreased in depression, patients do not necessarily have a higher propensity to fall asleep during the day, but rather experience a subjective state of sleepiness $[1,2]$. This state of sleepiness in depression differs from EDS

\footnotetext{
${ }^{*}$ Corresponding author. Tel.: +41 613255318; fax: +41613255577.

E-mail address: Christian.Cajochen@upkbs.ch (C. Cajochen).
}

as typically encountered in narcolepsy and obstructive sleep apnea (OSA). In the former, subjects experience EDS as "sleep attacks," namely short and recurrent periods of sleep in the daytime, regardless of the prior amount or quality of night sleep. In OSA, there is a higher propensity to fall asleep during the daytime, possibly due to sleep fragmentation and/or hypoxemia related to chronic intermittent respiratory events [3].

There is ample evidence for an association between daytime sleepiness and moderate to severe depression $[4,5]$. Given that insomnia, particularly when associated with impaired daytime function, plays a key role in the onset of depression [6], it is of significant clinical importance to unravel the link between EDS and depression. Furthermore, knowledge on the inter-relation between EDS and mood disorders may have ramifications for the treatment of depression $[7,8]$.

Apart from the sleep perspective, circadian rhythms play a key role in the association between depression and EDS. Depression is strongly intertwined with bio- 
logical rhythms on a wide range of dimensions, which includes neurobiological systems that underlie both the pathophysiology of depression, such as the serotonergic system, as well as the clinical aspects of depression [9]. This rhythmicity can manifest itself as an intra-episodic symptomatic expression - such as diurnal variation of mood and early morning awakening - or as an inter-episodic expression - such as seasonal recurrence of clinical depression or reappearance after therapeutic management. Disorders of the human circadian system per se can result in circadian misalignment, leading to sleep disturbances (namely, insomnia and/or EDS), reduced attention and impaired daytime alertness, lack of energy, lower performance, negative mood and gastrointestinal disorders. Interestingly, these symptoms also occur in depression, which further extends the idea that depression and circadian rhythms are intimately connected. Among depressive disorders, major depression, bipolar depression and seasonal affective disorder are defined as being both episodic and recurrent, thus possibly reflecting a chronobiological disorder [10]. Together with the rhythmicity of clinical symptoms, the aforementioned disorders have been associated with alterations of the rhythm of several physiological variables. These include sleep-wake cycle disruption and pathophysiological changes in the secretion of an array of hormones, including cortisol, TSH, GH and melatonin, among others [11-14].

The application of chronobiological and sleep regulatory concepts in the treatment of depressive disorders has led to effective therapeutic strategies, among which bright light therapy and sleep deprivation can be highlighted $[9,11]$. Furthermore, there is evidence that the circadian system is implicated in pharmacological treatment mechanisms, such as lithium therapy for bipolar depression [15]. Lithium can modify the phase-angle between circadian temperature rhythm and rest-activity cycle and lengthens the period of circadian rhythms by increasing the circadian period of firing rate rhythms of SCN neurons in a concentration-dependent manner $[16,17]$. Therefore, the stabilization of circadian rhythms may be a key action of clinically effective mood-stabilizing treatments.

In this review, studies on EDS and depression will be summarized and discussed within the framework of current sleep and chronobiology perspectives. A brief outlook of the probable chronobiological underpinnings will be given in order to provide insights on this inter-relationship and possibilities of chronobiological therapies.

\section{EDS and depression}

EDS is a common complaint in clinical practice [18]. On the societal level, the negative impacts of sleepiness are likewise significant. For instance, in the USA, a sizable proportion of adults ( $43 \%)$ report that they are so sleepy during the day that it interferes with their daily activities, with one out of five individuals experiencing this level of daytime sleepiness at least a few days per week or more [19]. On the individual level, EDS not only reduces personal efficiency of labour, but also leads to difficulties with concentration, memory and mood impairment, which further negatively impact performance and well being $[2,18,20]$.

EDS is commonly viewed as a cardinal sign of disturbed or inadequate sleep, and can be directly linked to depression $[4,5]$. In a recent study conducted with a random sample of 16,500 individuals in the USA, $8.7 \%$ displayed EDS [21]. Strikingly, EDS was more strongly associated with depression $(\mathrm{OR}=3.12)$ than with obesity or other metabolic factors and sleep-related breathing disorders. Indeed, depressive disorders are often associated with disrupted sleep. While the majority of depressed patients with sleep disruption suffer from insomnia, a considerable number of them report hypersomnia with concomitant daytime sleepiness [22]. This subset of patients is often diagnosed with bipolar depression or depression with DSM-IV-TR atypical features which include irritability, increased appetite and sleepiness [23].

Recently, we observed a significant association $(r=0.80)$ between EDS and suicidal ideation in depressed patients [24]. This unequivocal result was striking, considering that no specific relation of EDS with suicidal ideation had been previously described. An explanation for this finding may be that patients with severe depressive disorder rank daytime sleepiness in line with other major depressive symptoms [25-27]. If these findings are confirmed in a larger sample size, EDS should, therefore, be considered in the assessment of suicidal risk in depression.

Despite the significant evidence linking EDS to depression, the mechanisms underlying this relationship remain uncertain to date. One important line of research builds upon findings from several large-scale epidemiological studies, which have demonstrated that approximately $20 \%$ of depressed patients exhibit a frequently undiagnosed sleep-disordered breathing, mostly obstructive sleep apnea $[28,29]$. This goes in line with a large number of reports which have gathered data linking metabolic syndrome and, most importantly, obesity and diabetes or insulin resistance, together with elevated inflammatory markers, to both sleep-disordered breathing and depression [30-36].

\section{Depression and circadian rhythms}

Diurnal variations of mood and sleep disturbances belong to the core of classical symptoms that have linked depression to the circadian system [37]. Typical mood variation consists of a substantially improved mood in the evening in contrast to worsening in the 
morning after sleep [38]. The improved state in the evening includes other spheres, such as psychomotor activity and cognitive performance $[39,40]$. The existence of this type of daily variation is a predictor for positive response to certain types of treatment, such as sleep deprivation [41]. However, this pattern does not occur in all subtypes of depression and the opposite has also been described, particularly for bipolar depression and atypical depression [10]. In a given patient, different patterns of diurnal mood variation can occur during a single depressive episode.

Shortening of REM sleep latency and an abnormal distribution of REM sleep throughout the night may typically occur in depression [42-44]. In atypical depression, hypersomnia is frequent, with increased night sleep duration up to $12 \mathrm{~h}$, combined or not with EDS [24]. Since both REM sleep and sleep duration are under strong circadian control, these sleep alterations may reflect disturbances in the circadian system of depressed patients. There is a considerable body of evidence suggesting that daytime sleep may occur in depressed patients more frequently than commonly thought, possibly as a consequence of a dampened circadian rhythm of sleep propensity. In normal subjects, daytime napping can result in nocturnal sleep changes similar to those seen in depressed patients [1,21]. Thus, the nocturnal sleep disturbance in depression may actually reflect the occurrence of daytime naps with slow-wave sleep or slow-wave EEG activity [44].

Seasonal Affective Disorder (SAD) illustrates how an alteration between the external light-dark cycle (i.e., photoperiod) and circadian rhythms may elicit a depressive disorder. Most SAD patients become depressed in the winter time because of the later dawn, which leads their circadian rhythms to delay with respect to both clock time and to the sleep-wake cycle [45]. Changes in the latter sleep-wake are apparent, with most patients presenting hypersomnia in conjunction with EDS.

Abnormalities in the circadian rhythm are also observed in non-seasonal depressed patients, including variations in the circadian phase and amplitude of core body temperature $[9,38]$ and the endogenous melatonin rhythm [51,14]. Though overall studies have yielded conflicting results, chronobiological studies in which sleep timing and/or total amount of sleep were re-scheduled in patients provide evidence for the implication of circadian rhythms in depression $[46,47]$. However, it is still unclear whether these observations represent functional changes of the circadian system itself or are due to the influence of other factors downstream.

One critical aspect in major depression is the hyperactivity of hypothalamus-pituitary-adrenal (HPA) axis which reflects a stress response and/or abnormality of the circadian rhythm of cortisol [48]. Usually, the acrophase of the cortisol rhythm occurs early in the morning prior to awakening, while the nadir is registered at night, a short time after initiating sleep [12]. However, in depressive states, HPA hyperactivity leads to sustained high levels of cortisol resulting in insomnia and a hyperarousal state [13]. Furthermore, depressive patients often present high levels of thyrotropin-releasing hormone (TRH), and a reduction of the normal nocturnal elevation of serum thyrotropin has been suggested as a marker of an abnormal circadian rhythm of this hormone in depressive states [49]. As a consequence, hypothyroidism may be an additional factor for high levels of subjective sleepiness during daytime.

In addition to circadian and endocrine changes in depression, recent imaging studies have shed new light on the neurobiological basis of depression. The increased magnitude of ultradian (i.e., within-day) mood variability in depressed patients compared with nondepressed subjects [52] may be related to regional cerebral metabolic changes in the evening relative to morning and may reflect functional changes in components of the ventral and dorsal emotion neural systems [53]. Diurnal variation in mood may be related to different patterns of diurnal changes in the regional cerebral metabolic rate of glucose (rCMRglc) in depressed and healthy subjects. Morning-to-evening changes in rCMRglc differ with regard to the brain systems that promote wakefulness. In healthy adults, relative rCMRglc increases during the evening compared to the morning in wake-promoting brainstem and hypothalamic regions [54]. This pattern may reflect an input from the circadian timing system to promote wakefulness in face of the increasing sleep pressure. Considering that circadian rhythms can be attenuated in depression, diurnal variations in rCMRglc in brainstem and hypothalamic areas may be, therefore, blunted in depressed compared to healthy individuals [55].

\section{Sleepiness, mood and chronobiology: bridging the gap}

From a conceptual point of view, EDS can be considered to result from the biological need for sleep as characterized by a complex interaction between circadian and homeostatic processes [8]. Briefly, the master pacemaker driving circadian rhythms, the suprachiasmatic nuclei (SCN), is synchronized to the external light-dark cycle through retinal light input (light being the main synchronizer or "zeitgeber") [56]. A specialized nonvisual retinohypothalamic tract provides direct neuronal connection to the SCN from novel photoreceptors in the retinal ganglion cells that measure illuminance $[57,58]$. Although the SCN is the so-called master clock, circadian oscillators have been found in practically every cell [59]. Therefore, the SCN would act in a manner to organize an internal temporal conjunction of several peripheral oscillators. The homeostatic component is dependent on the duration of prior wakefulness and is dissipated during sleep, reinitiating its build-up during 
the following wake period [60]. The two-process model of sleep regulation has unified these two components to understand the timing and architecture of sleep [61]. This model can also be utilized to describe possible disturbances in either process during depression. The clinical sleep disturbance with early morning awakening could arise from an impaired build-up of S during waking (diminished sleep pressure) or an earlier timing of process C. Other possible abnormalities could lie in the decline of S during sleep or altered circadian period, phase, or amplitude [37].

Considering that mood is such a complex phenomenon, modulated by a plethora of factors extending from hormones to social situations, insight into its regulation is a rather difficult task. Surprisingly, though, mood is also determined by circadian and homeostatic factors $[62,63]$. Several hypotheses have been proposed to explain the mechanisms that underlie the relationship of sleep, sleep disorders and depression. According to the internal coincidence hypothesis, an abnormal relationship between the circadian system and the sleepwake cycle occurs in which the phase-angle between an advanced circadian pacemaker and the sleep-wake cycle is depressogenic [64]. In other words, depressive patients sleep at the wrong biological clock-time. Thus, such patients commonly develop insomnia that may be associated with EDS. On the other hand, the S-deficiency model proposes a deficient build-up of process $\mathrm{S}$ (i.e., sleep need) in depression, with process $\mathrm{C}$ remaining unaffected [60]. A third hypothesis suggests that disturbed neurochemical interaction mechanisms underlie abnormalities in the ultradian rhythm of REM sleep in depressed patients [37].

Two important protocols have been developed in order to dissect out the relative contributions of circadian and sleep homeostatic processes in humans, namely the constant routine and the forced desynchrony protocols [65]. In the first procedure, the amplitude and phase of many circadian rhythms can be elucidated without the masking effects of motor activity, meals and light conditions. In the second protocol, subjects live on artificially very long or very short days, so that the circadian system is no longer entrained to the imposed sleep-wake cycle, enabling the measurement of an individual's circadian period. The desynchronized subjects sleep at different circadian phases of the entire 24-h cycle, and subsequent analyses can differentiate the relative contribution of the sleep homeostat or the biological clock to a given variable [66]. Usually - but not always - both factors contribute substantially to subjective measures, such as mood, sleepiness and neurobehavioral performance $[67,68]$. However, only few studies have used these techniques in a controlled manner to unmask the contribution of the circadian clock in depression. These protocols can certainly contribute to a more accurate understanding of the circadian disruptions that occur in depression, and, thus, can be of substantial value in future research [9]. Table 1 gives an overview of constant routine and forced desynchrony studies in depressed patients $[69-72,67,73]$.

\section{Further hypotheses: sleepiness, neurotransmitters and hyperarousal}

Sleepiness may be deemed as a physiological state or urge, that promotes the onset of sleep, and which is reversed or satiated (although not always) by the attainment of adequate sleep. The neurobiological substrates of sleepiness are not understood completely. Sleepiness may reflect the waning of processes maintaining wakefulness or it may result from distinct neural systems acting to promote sleep [74]. In other words, these neurological substrates interact in a complex fashion to generate sleep and wakefulness. For instance, according to the "flip-flop" model of sleep and wakefulness [75], monoaminergic nuclei, including the locus coeruleus, the serotonergic dorsal and median raphé nuclei and the histaminergic tuberomammilary neurons, promote wakefulness by direct excitatory effects on the cortex and by inhibition of sleep promoting neurons of the ventrolateral preoptic nucleus. During sleep, the latter inhibits monoaminergic-mediated arousal regions through GABAergic and galaninergic projections. Orexin-containing neurons seem to play an important stabilizing role in the proposed "flip-flop" mechanism. On the other hand, the accumulation of a sleep-promoting substance that enhances the activity of sleep-promoting cells and reduces the activity of wake-promoting neurons may be a potential mechanism for homeostatic sleep regulation [75]. However, it remains to be determined how these and other brain structures act and interact to generate EDS in various disorders.

Circadian rhythms involve the same neurotransmitters that have been postulated to be important for depression, such as serotonin, thus leading to a situation in which changes in one system have repercussions on the other [9]. Serotonin plays an important part in sleep regulation, and prefrontal cortical serotonin has been linked to mood. All these inter-relationships have been conceptualized in a dual model whereby circadian rhythms and serotonin [76] together are involved in a system vulnerable to depression through genetic, hormonal and light exposure factors $[9,11,37,62,77]$.

From a pathophysiological standpoint, sleep disturbance in depressed patients with a chief complaint of daytime sleepiness appears to be associated with a centrally driven hyperarousal, whereas idiopathic sleepiness is usually associated with a centrally driven hypoarousal. This hypothesis has been supported by recent work in which both groups exhibited differences in night time sleep and daytime alertness [78]. The main postulation was that these disorders are related to the altered arou- 
Table 1

Constant routine (CR) and forced desynchrony (FD) protocols with depressed patients

\begin{tabular}{|c|c|c|c|c|c|c|}
\hline & $\begin{array}{l}\text { Type of } \\
\text { Study }\end{array}$ & Subjects & $\begin{array}{l}\text { Depression } \\
\text { diagnosis }\end{array}$ & Protocol procedure & Circadian marker & $\begin{array}{l}\text { Phase/amplitude/phase } \\
\text { angle changes } \\
\text { in depressed }\end{array}$ \\
\hline Dahl et al. (1993) [69] & $\mathrm{CR}$ & $6 \mathrm{SD}^{\mathrm{a}} 6 \mathrm{CT}^{\mathrm{b}}$ & SAD & 27-h CR & $\mathrm{DLMO}^{\mathrm{c}} \mathrm{CBT}^{\mathrm{d}}$ & $\begin{array}{l}\text { DLMO \& CBT } \\
\text { phase-delay }\end{array}$ \\
\hline Buysse et al. (1995) [70] & $\mathrm{CR}$ & $26 \mathrm{UP} 17 \mathrm{CT}$ & $\begin{array}{l}\text { DSM-IV } \\
\text { unipolar } \\
\text { depression }\end{array}$ & 36-h CR & $\begin{array}{l}\text { CBT melatonin } \\
\text { cortisol }\end{array}$ & No differences \\
\hline Wirz-Justice et al. (1995) [71] & $\mathrm{CR}$ & $11 \mathrm{SD} 8 \mathrm{CT}$ & SAD & 40-h CR & CBT melatonin & No differences \\
\hline Avery et al. (1997) [72] & $\mathrm{CR}$ & $6 \mathrm{SD} 6 \mathrm{CT}$ & SAD & 27-h CR & CBT cortisol & $\begin{array}{l}\text { CBT \& cortisol } \\
\text { phase-delay }\end{array}$ \\
\hline Koorengevel et al. (2002) [67] & FD & $7 \mathrm{SD} 7 \mathrm{CT}$ & SAD & 120-h FD (six 20-h days) & CBT melatonin & CBT amplitude $\downarrow$ \\
\hline Koorengevel et al. (2003) [73] & FD & $7 \mathrm{SD} 7 \mathrm{CT}$ & SAD & 120-h FD (six 20-h days) & CBT melatonin & CBT amplitude $\downarrow$ \\
\hline $\begin{array}{l}\text { a Seasonal depressed. } \\
\text { b Control subjects. } \\
\text { c Dim light melatonin onset. } \\
\text { d Core body temperature. }\end{array}$ & & & & & & \\
\hline
\end{tabular}

sal state and not sleep per se. This was further supported by the fact that idiopathic sleepiness is usually associated with dysfunction of the autonomic nervous system such as orthostatic hypotension and hypoactivity of the corticotropin-releasing hormone neuronal system (that is directly linked to arousal), whereas psychiatric hypersomnia is most frequently related to depression and anxiety, conditions deemed to be associated with hyperarousal [79]. However, more research on physiological parameters, such as metabolic rate and temperature, is required to test the hypothesis that sleepiness in depression, and idiopathic sleepiness are disorders of arousal.

\section{Diagnosis of EDS}

Although daytime sleepiness is highly prevalent, it is frequently under-diagnosed. To tackle this problem, the diagnosis of EDS should start with a precise definition and clinical history. EDS can result from the interaction of three following factors that account for increased sleepiness: physiological (due to the interplay of circadian and homeostatic components, as detailed above), manifest and introspective sleepiness [80]. Manifest sleepiness refers to the behavioural correlate of the physiological need for sleep. Reduced performance and alertness in a sleepy individual, involuntary sleepiness despite volitional attempts to remain awake and low performance in cognitive and behavioural tests can be some of the main hallmarks of "manifest sleepiness" in non-medicated depression. Manifest sleepiness is modulated by individual motivation and environmental factors. Introspective sleepiness consists of the individual ability to characterize internal subjective state [80]. In healthy subjects, there is an adequate perception of sleepiness after sustained sleep deprivation. Nevertheless, in chronic sleep deficit due to clinical disorders such as depression, the perception of sleepiness may be dramatically reduced, thus leading to an underestimation of sleepiness [6].

Depressed patients may present subjective complaints of tiredness or fatigue instead of more specific symptoms of sleepiness. In order to discriminate between tiredness and sleepiness, questions regarding the need to take naps, dozing in passive situations, or how long it is needed to initiate sleep at usual bedtime can help to distinguish real sleepiness from less specific complaints $[1,20,81]$. Problems concerning performance or neurocognitive function are often completely ignored. Performance alone may not always be the best indicator of sleepiness, as motivation may temporarily override a performance decrement. Compensatory strategies may also be invoked, such as avoiding increased errors by adopting a slower pace [82]. Likewise, individuals might also accept lower levels of achievement. An accurate evaluation of EDS usually requires several assessment methods [22]. A wide array of tools has been developed to assess sleepiness in a more objective fashion, although all of them have their shortcomings. Initially, introspective behavioural scales and performance tests were utilized to assess sleepiness. Subjective scales try to analyze the perception of alertness/sleepiness. However, a serious limitation to this approach is that patients must have an insight into their problem and be able to distinguish sleepiness from other factors affecting performance, which may not be the case. On the other hand, scales, such as the Stanford Sleepiness Scale and the Karolinska Sleepiness Scale, can convey an appropriate assessment of the momentary degree of alertness/sleepiness [83,84]. Furthermore, they are quite useful in tracking symptoms over time, although they 
do not provide a global perspective of sleepiness. The Epworth Sleepiness Scale offers a better method for assessing overall sleepiness, since it reports on sleepiness over a one-month period, including both active and passive situations of possible behavioural sleepiness [85]. Visual analogue scales require the subject to provide a response along a linear $100 \mathrm{~mm}$ line, ranging from very sleepy to very alert [6]. The main advantage of these scales is that they can be utilized often and sequentially to track subtle changes in the feeling of sleepiness over a given time period. Performance tests, such as the PVT, have been frequently utilized to measure sustained attention [86]. However, questionnaires utilized in the assessment of subjective sleepiness (e.g., Epworth or Stanford scales) do not always correlate with objective quantifications. Objective tests of daytime sleepiness rely on measurement of physiological parameters, the most extensively used test being the multiple sleep latency test (MSLT). The MSLT is performed after an overnight polysomnogram in order to assess sleep during the prior night (which controls for nocturnal sleep disruption that could account for daytime symptoms, such as obstructive sleep apnea) [87]. Accordingly, mean sleep latency $>10 \mathrm{~min}$ is deemed normal, the $5-10 \mathrm{~min}$ range as a grey zone and $<5 \mathrm{~min}$ as severe daytime sleepiness.

Waking EEG changes may also provide insight into the state of sleepiness in depression. For instance, in a 40-h constant routine study looking at the EEG and subjective sleepiness in seasonal affective disorder (SAD), we observed that the progressive build-up of sleepiness was considerably reduced in SAD patients [88]. The time course of waking EEG theta-alpha activity showed a rapid increase during the first $10 \mathrm{~h}$, which then remained constant, in contrast to control subjects who showed a slower continuous exponential increase in the course of the 40-h episode of sustained wakefulness. Given that this pattern was independent of clinical state or season, SAD patients may have a trait deficiency in the homeostatic build-up of sleep pressure.

\section{Chronobiological treatments for sleepiness and depression}

A chronobiological approach may address some of the unmet needs in the treatment of depression, such as diminishing the latency of onset of antidepressant action, reducing residual symptoms and preventing relapse in the long-term. Circadian rhythm and sleep research have led to promising non-pharmacological therapies $[9,11,89]$. Sleep deprivation therapy exerts a rapid and dramatic, albeit usually short-lasting, improvement of mood in the majority of patients with major depressive disorder. Light therapy has been applied to a wide range of mood disorders as standalone therapy or as an adjunct to antidepressant pharmacotherapy [90]. Light acts as a synchronizing agent for disturbed circadian rhythms, while sleep deprivation challenges the sleep-wake homeostat [91,92]. Table 2 summarizes the main chronobiological therapies employed for depressive disorders.

Total sleep deprivation (TSD) for one whole night improves depressive symptoms in $40-60 \%$ of treatments $[37,92]$. The degree of clinical change spans a continuum from complete remission to worsening. Sleep deprivation (SD) response shows up in the SD night or on the following day. After recovery sleep, $50-80 \%$ of first day responders suffer a complete or partial relapse; nevertheless, improvement can last for weeks. Sleep seems to lead to the relapse, although this may not necessarily be the case. Treatment effects may be stabilized by antidepressant drugs, lithium, shifting of sleep time or light therapy [93]. Current opinion is that partial sleep deprivation (PSD) in the second half of the night is equally effective as TSD. Sleep deprivation may hasten the onset of antidepressant medication action and repeated trials of SD can also be an efficient treatment strategy in drug refractory depression $[37,91,92]$. Given that insomnia associated or not with EDS is such a prominent feature of depression, further investigations may consider track-

Table 2

Chronobiological therapies of depressive disorders

\begin{tabular}{|c|c|c|}
\hline & Therapeutic duration & Duration of response \\
\hline Total sleep deprivation (TSD) & Hours & 1 day \\
\hline Partial sleep deprivation (PSD) in the second half of the night & Hours & 1 day \\
\hline Repeated TSD or PSD & Hours & Days/weeks \\
\hline Repeated TSD or PSD with antidepressant therapy & Hours & Weeks/months \\
\hline Sleep-wake cycle phase advance & 3 days & Up to 2 weeks \\
\hline TSD followed by sleep phase advance & Hours & Up to 2 weeks \\
\hline Single or repeated TSD or PSD followed by light therapy & Hours & Weeks/months \\
\hline Single or repeated TSD or PSD followed by phase advance and light therapy & Hours & Weeks/months \\
\hline Single or repeated TSD or PSD combined with lithium or serotonin selective receptor inhibitors & Hours & Months \\
\hline Light therapy (winter seasonal MDD) & Days & Weeks/months \\
\hline Light therapy (non-seasonal MDD) & Weeks & Weeks/months \\
\hline Light therapy combined with serotonin selective reuptake inhibitors (non-seasonal MDD) & Up to 2 weeks & Weeks/months \\
\hline
\end{tabular}

*Modified by Wirz-Justice et al. (2005) [81].

MDD, major depressive disorder. 
ing EDS as a concomitant outcome measure of sleep deprivation therapy for depression.

Light per se exerts powerful non-visual effects on a wide range of biological functions including the modulation of human alertness levels. Factors such as the dose (illuminance levels), exposure duration, timing and wavelength of light have important repercussions on the alerting response to light in humans and can lead to a substantial decrement in sleepiness [94]. Bright light therapy is characterized by a fast onset of antidepressant action and by additive properties to antidepressant medication [95]. Bright light therapy, beginning in the morning after partial sleep deprivation, has been demonstrated to prevent depressive relapse after recovery sleep in sleep deprivation responders [95]. Although until now bright light therapy has been regarded as a first-line treatment only for seasonal affective disorder (SAD) [96], recent studies on non-seasonal depression and the Cochrane meta-analysis on light therapies for this type of depression have provided evidence for the efficacy of bright light as an adjuvant to antidepressant pharmacotherapy $[94,97,95,98]$. Taking into account the insufficient remission rate of depression solely with pharmacotherapy and the favourable risk-to-benefit ratio of light therapy, it can offer a promising non-pharmacological biologically oriented treatment approach [90].

The combination of treatment strategies that manipulate circadian rhythms has lead to some very promising results. A strategy to block relapse in successful sleep deprivation responders includes therapeutic interventions combining sleep deprivation, phase advance and light therapy. For instance, one combination encompasses total sleep deprivation plus phase advance [44]. The underlying assumption is that, after a successful sleep deprivation, the avoidance of recovery sleep during a hypothesized critical early morning time period may prevent sleep deprivation associated relapses, with a concomitant improvement in both mood and subjective sleepiness [99].

On the pharmacological domain, the use of modafinil, a wake-promoting agent, has been recently suggested as a well-tolerated adjuvant in the treatment of EDS and depression, with a substantial improvement in both mood and sleepiness [100]. Furthermore, it is a particularly attractive alternative to other stimulants because of its low abuse potential. However, in a double-blind placebo control study, no significant improvements for daytime sleepiness, depression scores or overall clinical state were found [101]. Thus, caution should be kept in mind when using modafinil in depression.

Regarding antidepressants, some of the therapeutic properties of selective serotonin (5-HT) reuptake-inhibiting (SSRI) drugs can be related to the $5-\mathrm{HT}_{2 \mathrm{C}}$ receptor subtype, and it is interesting to observe that $5-\mathrm{HT}_{2 \mathrm{C}}$ receptor agonists in the rat $\mathrm{SCN}$ mimic the effects of light [50]. Serotonergic drugs may thus improve entrainment.

Recently, a novel antidepressant drug, agomelatine, a melatonin agonist and 5- $\mathrm{HT}_{2 \mathrm{c}}$ antagonist, targeted both the circadian system and mood. Agomelatine [102] has identical physiological actions to melatonin [103] when administered in the evening to advance circadian phase and to promote a rapid sleep onset without after-effects on the following day. Indeed, unlike other antidepressants, notably SSRIs, agomelatine does not lead to insomnia, fragmentation of slow-wave sleep and nightmares, but rather improves the quality of sleep, with possible increases in both slow-wave sleep, and to a lower extent, REM sleep in depressed patients [104]. The interesting aspect of this treatment is that its core action aims at the circadian system, and it has been shown to be especially useful for synchronizing circadian rhythms of body temperature, cortisol and thyroid-stimulating hormone secretion [102]. Considering this, agomelatine can be indicated for the management of depression associated with EDS.

Recently, orexin-antagonists Ox1 and Ox2 receptors are being tested in the treatment of insomnia. Orexins are hypothalamic peptides that may play an important role in maintaining wakefulness in mammals. When administered during the active period of the circadian cycle, a dual antagonist of both orexin OX1 and OX2 receptors was shown to increase subjective and objective EEG sleep in humans [105]. Although this study targeted narcolepsy, these results may open new perspectives for investigating the role of endogenous orexins in sleep-wake regulation. Given the central role played by orexins in regulating sleep-wake cycles, it has been suggested that several sleep disorders may be associated with maladaptive orexinergic tone [105]. Whether orexin-antagonists can be utilized for other types of sleep disturbances, such as insomnia in depressed patients, however, remains to be answered.

\section{Conclusions}

EDS is a complaint frequently encountered in depression. Notwithstanding the significant evidence that links EDS to depression, the underlying mechanisms of this relationship remain uncertain. Considering that depression is intertwined with circadian rhythms on a wide array of dimensions, a chronobiological approach may shed new light on the complex interaction of EDS and depression and offer promising therapeutic strategies.

\section{References}

[1] Fava M. Daytime sleepiness and insomnia as correlates of depression. J Clin Psychiatry 2004;65:27-32. 
[2] Young TB. Epidemiology of daytime sleepiness: definitions, symptomatology and prevalence. J Clin Psychiatry 2004;65: 12-6.

[3] Colt HG, Haas H, Rich GB. Hypoxemia vs sleep fragmentation as cause of EDS in obstructive sleep apnea. Chest 1992;100:1542-8.

[4] Breslau N, Roth T, Rosenthal L, Andreski P. Sleep disturbance and psychiatric disorders: a longitudinal epidemiological study of young adults. Biol Psychiatry 1996;39:411-8.

[5] Hublin C, Kapiro J, Partinen M, Heikkilä K, Koskenvuo M. Daytime sleepiness in an adult Finnish population. J Intern Med 1996;239:417-23.

[6] Ohayon MM, Roth T. Place of chronic insomnia in the course of depressive and anxiety disorders. J Psychiat Res 2003;37:9-15.

[7] Breslau N, Roth T, Rosenthal L, Andreski P. Daytime sleepiness: an epidemiological study of young adults. Am J Public Health 1997;87:1649-53.

[8] Tylee A, Gastpar M, Lepine JP, Mendlewicz J. DEPRESS II (Depression Research in European Society II): a patient survey of the symptoms, disability and current management of depression in the community. DEPRESS Steerling Committee. Int Clin Psychopharmacol 1999;14:139-51.

[9] Wirz-Justice A. Chronobiology and mood disorders. Dialog Clin Neurosci 2003;5:315-25.

[10] Cano-Lozano MC, Espinosa-Fernandez L, Miro E, Buela-Casal G. A review of sleep disorders in depression. Rev Neurol 2003;36:366-75

[11] Wirz-Justice A. Circadian disturbances in depression: therapeutic perspectives. Medicographia 2003;25:29-36.

[12] Stokes PE. The potential role of excessive cortisol induced by HPA hyperfunction in the pathogenesis of depression. Eur Neuropsychopharmacol 1996;5:77-82.

[13] Schule C, Baghai T, Zwanzger P, Minov C, Padberg F, Rupprecht R. Sleep deprivation and hypothalamic-pituitaryadrenal (HPA) axis activity in depressed patients. J Psychiatr Res 2001;35:239-47.

[14] Post RM. Adjunctive strategies in the treatment of refractory bipolar depression: clinician options in the absence of a systematic database. Pharmacotherapy 2005;6:531-46.

[15] Duncan WC. Circadian rhythms and the pharmacology of affective illness. Pharamacol Ther 1996;71:253-312.

[16] Nagayama H. Chronic administration of imipramine and lithium changes the phase-angle relationship between activity and core body temperature circadian rhythms. Chronobiol Int 1996;13:251-9.

[17] Abe M, Herzog ED, Block GD. Lithium lengthens the circadian period of individual suprachiasmatic nucleus neurons. Neuroreport 2000;28(11):3261-4.

[18] Ohayon MM, Caulet M, Philip P. How sleep and mental disorders are related to daytime sleepiness. Arch Intern Med 1997; 157:2645-52.

[19] National Institutes of Health State of the Science State Conference. Sleep 2005;28:1049-1057.

[20] Guilleminault C, Brooks SN. EDS - a challenge for the practising neurologist. Brain 2001;124:1482-91.

[21] Bixler EO, Vgontzas AN, Lin HM, Calhoun SL, Vela-Bueno A, Kales A. EDS in a general population sample: the role of sleep apnea, age, obesity, diabetes, and depression. J Clin Endoc Metabol 2005;90:4510-5.

[22] Bittencourt LRA, Silva RS, Santos RF, Pires MLN, Mello MT. Excessive daytime sleepiness. Rev Bras Psiquiatr 2005;27: 16-21.

[23] American Psychiatric Association. Diagnostic and statistical manual of mental disorders (DSM-IV-TR). Washington (DC): American Psychiatric Press; 2000.

[24] Chellappa SL, Araújo JF. EDS in patients with depressive disorder. Rev Bras Psiquiatr 2006;28:126-30.
[25] Chellappa SL, Araujo JF. Sleep disorders and suicidal ideation in patients with depressive disorder. Psychiatr Res 2007;153:131-6.

[26] Schmidtke A, Bille-Brahe U, De Leo D, Kerkhof A, Wasserman $\mathrm{D}$, editors. Suicidal behaviour in Europe: results from the WHO/ EURO Multicentre Study on Suicidal Behaviour. Göttingen, Germany: Hogrefe \& Huber; 2001.

[27] Van Heeringen K. The neurobiology of suicide and suicidality. Can J Psychiatry 2003;48:292-300.

[28] Ohayon MM. The effects of breathing-related sleep disorders on mood disturbances in the general population. J Clin Psychiatry 2003;64:1195-2000.

[29] Sharafkhaneh A, Giray N, Richardson P, Young T, Hirshkowitz M. Association of psychiatric disorders and sleep apnea in a large cohort. Sleep 2005;28:1405-11.

[30] Schuster SR, Tabba M, Sahota P. Relationship between the cardiometabolic syndrome and obstructive sleep apnea. J Cardio Metabolic Syndrome 2006;1:204-8.

[31] Schröder CM, O'Hara R. Depression and obstructive sleep apnea (OSA). Ann Gen Psychiatry 2005;4:13. doi:10.1186/1744859X-4-13.

[32] Gami AS, Somers VK. Obstructive sleep apnea, metabolic syndrome, and cardiovascular outcomes. Eur Heart J 2004;25:709-11.

[33] Lett HS, Blumenthal JA, Babyak MA, Sherwood A, Strauman T, Robins C, et al. Depression as a risk factor for coronary artery disease: evidence mechanisms and treatment. Psychosom Med 2004;66:305-15.

[34] Raikkonen K, Matthews KA, Kuller LH. The relationship between psychological risk attributes and the metabolic syndrome in healthy women: antecedent or consequence? Metabolism 2002;51:1573-7.

[35] McCaffery JM, Niaura R, Todaro JF, Swan GE, Carmelli D. Depressive symptoms and metabolic risk in adult male twins enrolled in the National Heart, Lung, and Blood Institute twin study. Psychosom Med 2003;65:490-7.

[36] Ramasubbu R. Insulin resistance: a metabolic link between depressive disorder and atherosclerotic vascular diseases. Med Hypotheses 2002;59:537-51.

[37] Wirz-Justice A, Van den Hoofdakker RH. Sleep deprivation in depression: what do we know, where do we go? Biol Psychiatry 1999;46:445-53.

[38] Boivin DB. Influence of sleep-wake and circadian rhythm disturbances in psychiatric disorders. J Psychiatry Neurosci 2000;25:446-58.

[39] Murray G. Diurnal mood variation in depression: a signal of disturbed circadian function? J Affect Disord 2007;102: 47-53.

[40] Van den Hoofdakker RH. Chronobiological theories of nonseasonal affective disorders and their implications for treatment. $\mathrm{J}$ Biol Rhythms 1994;9:157-83.

[41] Gordijn MCM, Beersma DGM, Bouhuys AL, Reinink E, Van den Hoofdakker RH. A longitudinal study of diurnal mood variation in depression: characteristics and significance. J Affect Disord 1994;31:261-73.

[42] Ohayon MM. Epidemiology of insomnia: what we know and what we still need to learn. Sleep Med Rev 2000;6:97-111.

[43] Thase ME, Fasicka AL, Berman SR, Simon AD, Reynolds CF. Electroencephalographic sleep profiles in single-episode and recurrent unipolar forms of major depression: comparison during acute depressive states. Biol Psychiatry 1995;38: 99-108.

[44] Riemann D, Berger M, Voderholzer U. Sleep and depression results from psychobiological studies: an overview. Biol Psychology 2001;57:67-103.

[45] Lewy AJ, Lefler BJ, Emens JS, Bauer VK. The circadian basis of winter depression. Proc Nat Acad Sciences 2006;103:7414-9. 
[46] Bouhuys AL. Towards a model of mood responses to sleep deprivation in depressed patients. Biol Psychiatry 1991;29:600-12.

[47] Berger M, Vollmann J, Hohagen F, Konig A, Lohner H, Voderholzer U, et al. Sleep deprivation combined with consecutive sleep phase advance as a fast-acting therapy in depression: an open pilot trial in medicated and unmedicated patients. Am J Psychiatry 1997;154:870-2.

[48] Steiger A. Sleep and the hypothalamo-pituitary-adrenocortical system. Sleep Med Rev 2002;6:125-38.

[49] Miller AH. Hypothyroidism in major depression: relevance to pathophysiology and treatment. Biol Psychiatry 2001;49:391-404.

[50] Cajochen C, Kräuchi K, Wirz-Justice A. Role of melatonin in the regulation of human circadian rhythms and sleep. J Neuroendocrinol 2003;15:432-7.

[51] Lewy AJ. Clinical applications of melatonin in circadian disorders. Dialogues Clin Neurosci 2003;5:399-413.

[52] Wefelmeyer T, Kuhs H. Diurnal mood variation in melancholic patients and healthy controls. Psychopathology 1996;29:184-92.

[53] Phillips ML, Drevets WC, Rauch SL, Lane R. Neurobiology of emotion perception I: the neural basis of normal emotion perception. Biol Psychiatry 2003;54:504-14.

[54] Buysse DJ, Nofzinger EA, Germain A, Meltzer CC, Wood A, Ombao H. Regional brain glucose metabolism during morning and evening wakefulness in humans: preliminary findings. Sleep 2004;27:1245-54.

[55] Germain A, Nofzinger EA, Meltzer CC, Wood A, Kupfer DJ, Moore RY, Buysse DJ. Diurnal variation in regional brain glucose metabolism in depression. Biol Psychiatry 2007;62:438-45.

[56] Klein DC, Moore RY, Reppert SM. Suprachiasmatic nucleus: the mind's clock. New York: Oxford University Press; 1991.

[57] Berson DM, Dunn FA, Takao M. Phototransduction by retinal ganglion cells that set the circadian clock. Science 2002;295:1070-3.

[58] Provencio I, Rodriguez IR, Jiang G, Hayes WP, Moreira EF, Rollag MD. A novel human opsin in the inner retina. J Neurosci 2000;20:600-5.

[59] Schibler U, Ripperger J, Brown SA. Peripheral circadian oscillators in mammals: time and food. J Biol Rhythms 2003;18:250-60.

[60] Borbely AA, Wirz-Justice A. Sleep, sleep deprivation and depression. Human Neurobiol 1982;1:205-10.

[61] Daan S, Beersma DGM, Borbely AA. Timing of human sleep: recovery process gated by a circadian pacemaker. Am J Physiol Regul Integr Comp Physiol 1984;246:161-83.

[62] Wirz-Justice A. Biological rhythm disturbances in mood disorders. Int Clin Psychopharmacol 2006;21:S11-5.

[63] Boivin DB, Czeisler CA, Dijk DJ, Duffy JF, Folkard S, Minors DS, et al. Complex interaction of the sleep-wake cycle and circadian phase modulates mood in healthy subjects. Arch Gen Psychiatry 1997;54:145-52.

[64] Wehr TA, Wirz-Justice A. Internal coincidence model for sleep deprivation and depression. In: Koella WP, editor. Sleep. Karger: Basel; 1981.

[65] Duffy JF, Dijk DJ. Getting through to circadian oscillators: why use constant routines? J Biol Rhythms 2002;17:4-13.

[66] Dijk DJ, Shanahan TL, Duffy JF, Ronda JM, Czeisler CA. Variation of electroencephalographic activity during non-rapid eye movement and rapid eye movement sleep with phase of circadian melatonin rhythm in humans. J Physiol 1997;505:851-8.

[67] Koorengevel KM, Beersma DG, den Boer JA, van den Hoofdakker RH. Mood regulation in seasonal affective disorder patients and healthy controls studied in forced desynchrony. Psychiatry Res 2003;117:57-74.
[68] Cajochen C, Khalsa SBS, Wyatt JK, Czeisler CA, Dijk DJ. EEG and ocular correlates of circadian melatonin phase and human performance decrements during sleep loss. Am J Physiol Regul Integr Comp Physiol 1999;277:640-9.

[69] Dahl K, Avery DH, Lewy AJ, Savage MV, Brengelmann GL, Larsen LH, et al. Dim light melatonin onset and circadian temperature during a constant routine in hypersomnic winter depression. Acta Psychiatr Scand 1993;88:60-6.

[70] Buysse DJ, Monk TH, Kupfer DJ, Frank E, Stapf D. Circadian patterns of unintended sleep episodes during a constant routine in remitted depressed patients. J psychiat Res 1995;29:407-16.

[71] Wirz-Justice A, Kräuchi K, Brunner DP, Graw P, Haug HJ, Leonardt $\mathrm{G}$, et al. Circadian rhythms and sleep regulation in seasonal affective disorder. Acta Neuropsychiatr 1995;7:41-3.

[72] Avery DH, Larsen LH, Prinz PN, Dahl K, Savage MV, Brengelmann GL. Circadian temperature and cortisol rhythms a constant routine are phase-delayed in hypersomnic winter depression. Biol Psychiatry 1997;41:1109-23.

[73] Koorengevel KM, Beersma DG, den Boer JA, van den Hoofdakker RH. A forced desynchrony study of circadian pacemaker characteristics in seasonal affective disorder. J Biol Rhythms 2002;17:463-75.

[74] McCarley RW. Sleep neurophysiology: basic mechanisms underlying control of wakefulness and sleep. In: Chokroverty $\mathrm{S}$, editor. Sleep disorders medicine: basic science technical considerations and clinical aspects. Boston: Butterworth-Heinemann; 1999. p. 21-50.

[75] Saper CB, Chou TC, Scammell TE. The sleep switch: hypothalamic control of sleep and wakefulness. Trends Neurosci 2001;24:726-31.

[76] Lam RW, Tam EM, Yatham LN, Shiah IS, Zis AP. Seasonal depression: the dual vulnerability hypothesis revisited. J Affect Disord 2001;63:123-32.

[77] Southmayd SE, Cairns J, David MM. Sleep disturbance in depression reconsidered. Can J Psychiatry 1991;36:366-73.

[78] Vgontzas AN, Bixler EO, Kales A, Criley C, Vela-Bueno A. Differences in nocturnal and daytime sleep between primary and psychiatric hypersomnia: diagnostic and treatment Implications. Psychosomatic Med 2000;62:220-6.

[79] Guilleminault C, Stoohs R, Clerk A, Cetel M, Maistros P. A cause of excessive daytime. Sleep 1993;104:781-7.

[80] Gupta RM. Approach to the sleepy patient. Med Health 2002;85:86-9.

[81] Pigeon WR, Sateia MJ, Ferguson RJ. Distinguishing between EDS and fatigue: toward improved detection and treatment. J. Psychosom Res 2003;54:61-9.

[82] Dorrian J, Rogers N, Dinges D. Psychomotor vigilance performance: neurocognitive assay sensitive to sleep loss. Sleep deprivation: clinical issues, pharmacology and sleep loss effects. New York: Marcel Dekker; 2005.

[83] Hoddes E, Dement W, Zarcone V. The development and use of the Stanford sleepiness scale. Psychophysiology 1972;9:150.

[84] Akerstedt T. Wide awake at odd hours. Stockholm: Swedish Council for Work Life Research; 1996.

[85] Johns MW. Sleepiness in different situations measured with the Epworth Sleepiness Scale. Sleep 1991;17:703-10.

[86] Blatter K, Cajochen C. Circadian rhythms in cognitive performance: methodological constraints, protocols, theoretical underpinnings. Physiol Behavior 2007;90:196-208.

[87] Carskadon MA, Dement WC, Mitler MM, Roth T, Westbrook PR, Keenan S. Guidelines for the multiple sleep latency test (MSLT): a standard measure of sleepiness. Sleep 1986;9:519-24.

[88] Cajochen C, Brunner DP, Krauchi K, Graw P, Wirz-Justice A. EEG and subjective sleepiness during extended wakefulness in seasonal affective disorder: circadian and homeostatic influences. Biol Psychiatry 2000;47:610-7. 
[89] Terman M, Terman JS. Bright light therapy: side effects and benefits across the symptom spectrum. J Clin Psychiatry 1999;60:799-808.

[90] Even C, Schröder CM, Friedman S, Rouillon F. Efficacy of light therapy in nonseasonal depression: a systematic review. J Affect Disord 2007. doi:10.1016/j.jad.2007.09.008..

[91] Wirz-Justice A, Benedetti F, Berger M, Lam RW, Martiny K, Terman M, et al. Chronotherapeutics (light and wake therapy) in affective disorders. Psychol Medicine 2005;35:939-44.

[92] Giedke H, Schwarzler F. Therapeutic use of sleep deprivation in depression. Sleep Med Rev 2002;6:361-77.

[93] Benedetti F, Barbini B, Fulgosi MC. Combined total sleep deprivation and light therapy in the treatment of drug-resistant bipolar depression: acute response and long-term remission rates. J Clin Psychiatry 2005;66:1535-40.

[94] Cajochen C. Alerting effects of light. Sleep Med Rev 2007;11:453-64.

[95] Loving RT, Kripke DF, Shuchter SR. Bright light augments antidepressant effects of medication and wake therapy. Depress Anxiety 2002;16:1-3.

[96] Lam RW, Levitan RD. Pathophysiology of seasonal affective disorder: a review. J Psychiatry Neurosci 2000;25:469-80.

[97] Martiny K, Lunde M, Unden M, Dam H, Bech P. Adjunctive bright light in non-seasonal major depression: results from clinician-rated depression scales. Acta Psychiatr Scand 2005;112:117-25.

[98] Tuunainen A, Kripke DF, Endo T. Light therapy for nonseasonal depression (Cochrane Review). In: The Cochrane Library, Chichester, UK: Wiley \& Sons, Ltd.2004.
[99] Berger M, Vollmann J, Hohagen F, Konig A, Lohner H, Voderholzer U, Riemann D. Sleep deprivation combined with consecutive sleep phase advance as a fast-acting therapy in depression. Am J Psychiatry 1997;154:870-2.

[100] DeBatista C, Lambke A, Solvason HB. A prospective trial of modafinil as an adjunctive treatment of major depression. J Clin Psychopharmacol 2004;24:87-90.

[101] DeBatista C, Doghramji K, Menza MA. Adjuvant modafinil for the short-term treatment of fatigue and sleepiness in patients with major depressive disorder: A preliminary double-blind, placebo-controlled study. J Clin Psychiatry 2003;64: $1057-64$.

[102] Loo H, D'haenen H, Hale A. Determination of the dose of agomelatine, a melatoninergic agonist and selective 5-HT2c antagonist, in the treatment of major depressive disorder: a placebo-controlled dose range study. Int $\mathbf{J}$ Neuropsychopharmacol 2002;17:239-47.

[103] Cajochen C, Krauchi K, Mori D, Graw P, Wirz-Justice A. Melatonin and S-20098 increase REM sleep and wake-up propensity without modifying NREM sleep homeostasis. Am J Physiol Regul Integr Comp Physiol 1997;272:1189-96.

[104] Hamon M, Bourgoin S. Pharmacological profile of antidepressants: a likely basis for their efficacy and side effects? Eur Neuropsychopharmacol 2006;16:625-32.

[105] Brisbare-Roch C, Dingemanse J, Koberstein R, Hoever P, Aissaoui H, Flores S. Promotion of sleep by targeting the orexin system in rats, dogs and humans. Nat Med 2007;12: $150-5$. 